\title{
Research of the Data Security Related Issues in Cloud Computing Services
}

\author{
$\mathrm{Nan} \mathrm{Hu}^{1, \mathrm{a}}$ \\ ${ }^{1}$ Laoning Institute of Science and Technology, Benxi \\ hunter2011@foxmail.com
}

Keywords: Cloud Computing Services, Data Security

\begin{abstract}
Cloud computing is a kind of data-intensive computing and it has a great advantage in the data storage, data calculation and data transmission. But in the practical application, the problems of data security of cloud computing is worrying, the security problem of centralized data storage and transmission on the network is not fully solved, this is also a challenge for the cloud computing services. This paper detailed researches and analyzes the present situation about cloud computing services, and puts forward the basic strategy of data security in cloud computing services to improve the security of cloud computing data.
\end{abstract}

\section{Introduction}

With the rapid development of modern network technology, cloud computing technology as an emerging network technology research and applications, it has attracted more and more people's attention. However, the security issues and efficiency of cloud computing have become the key issues that constraint the rapid development and promotion of cloud computing, this paper focuses on cloud computing security issues associated with performance issues, and give the kind of cloud computing services security mechanisms on this basis [1].

Cloud computing is a new modern network service model. Cloud computing is actually a concept in the IT industry under the social division of labor. Cloud computing is Grid Computing, Distributed Computing, Parallel Computing, Utility Computing, Network Storage Technologies, Virtualization, Load Balance and other traditional techniques combined into and it is the latest computer network services model. In such a network service model, the way the users and businesses using and working with networks have undergone enormous changes. The most typical change is in the before users and business are often thinking about purchasing products at the first time, and after the emergence of cloud computing, they only need to purchase services. In the course of their work users and enterprises are totally unnecessary to buy variety of hardware and software systems, and even they do not need to care about whether the room has a power phenomenon, whether the air conditioning was not functioning properly, whether specialized maintenance personnel due diligence, etc. and they do not need to worry about in the project implementation process for the acquisition of hardware and software. They do just is paying related costs to cloud computing service providers, and then they get the service they want.

Cloud computing covers all levels from the underlying infrastructure to the uppermost layer of the application, involving servers, networking, storage, security, IT systems management. From the entire network technology and the computer industry analysis, cloud computing is completely different from the previous business model, the propose and promote of cloud computing will lead to a new explosive growth of the whole industry. In this new business model, eventually it will be able to make the user computing, software applications, data storage management services as a very common facilities and services, just like modern people use gas, electricity and water as usual. While research facing the cloud computing has become one of the current research focus. 


\section{The Outline of Cloud Computing Technology}

The definition. As a new form of service - Cloud computing has no precise and uniform definition. The international generally accepted definition is: Cloud computing is a pay-per-use model that supplies available, convenient, on-demand network access into a shared pool of configurable computing resources, and these resources can be quickly offered, simply need minimal management effort or have very little interaction with service provider. Data of cloud computing has six stages, namely, data generation, migration, use, sharing, storage, destruction [2].

The features. Compared with traditional enterprise data storage and processing, cloud computing services are with low cost, fast, and flexible. Besides cloud computing services as well as on-demand services, convenient time, resource sharing, based virtualization technology, reducing the burden on the user terminal processing, and reduce dependence on the expertise and other features. You can also optimize resource, cost savings for enterprises to bring economic benefits.

The applications. As the technology matures, cloud computing has been applied to many fields, including medicine and medical fields, manufacturing, finance and energy sectors, e-government, education and scientific research fields, the field of telecommunications, the future may also covers all aspects of society .

\section{The Data Security in Cloud Computing Services}

Cloud computing services bring convenient to the enterprise, it is also facing several threats, such as data corruption, data loss, account hijacking, abuse and malicious use, inadequate review and share technical problems.

The security of data storage. Cloud computing meets the variety needs of the user by centrally storing data, this model is very easy endanger the security of user data. For users, the security of user's data has relationship with the major decision, and it is the premise and basis for user decision, therefore, data security has become one of the biggest obstacles to the development of cloud computing. In cloud computing, data storage is provided in the form of service, for the cloud provider, the data is transparent, and the data stored on Internet servers, which increases the security of data transmission [3].

The privacy of data. Cloud computing services are also involved in the privacy of the data users need, how to ensure the data privacy stored in the cloud computing service providers is not in the illegal use is the user care. Users find service in the cloud service according $t$ their needs, which requires involving the privacy of users. Users making some decisions, you need data to provide a basis for decision-making, the data on the other hand reflects the user's next dynamic. If these data are the illegal use, particularly its competitors, companies may lose surprise, preemptive opportunities, resulting in loss of customers, so the privacy of data security relationship with the economic interests of users. And there are many well-known companies were accused of leaking user privacy.

The storage area of data is not clear. Because cloud environments is without geographical restrictions, so the data are likely to be stored in any place in the world of data storage centers, the legal aspects of jurisdiction is not yet clear, the data stored in other countries are likely to be occupied by other countries. Some business users cannot obtain compensation through the existing national laws when important data is lost or misappropriated. Imagine what being misappropriated is trade secrets, such as when product information, operational data and other data, it will make companies less competitive and the losses are indelible.

There are many security risks in Internet. Application characteristics of cloud computing ubiquitous, users can access data through the network at any time. But the current Internet, there are many security risks, such as hacking, virus implanted Trojans vandalism, bugs, account hijacking and unsafe API like. Internet security risks have greatly reduced the data security of cloud computing which collected network environment. 


\section{The Strategies on the Data Security in Cloud Computing Services}

Improve relevant policies. The survival and development of cloud computing services are inseparable from social background and any new things are the result of social development. Comply came into being with the development of cloud computing era, in turn, society should provide some support and protection for the development of cloud computing. In the current issue of data security has become the main obstacle to the development of cloud computing services, in addition to improving technology, strengthen between providers and users of cloud computing constraints, it should also improve the macro-environment, namely improving the policy on cloud computing services . Cloud computing is regarded as the third wave of information technology, the state should seize the opportunity to strengthen the cloud computing to support efforts to develop active industrial development policies and regulations, to build a relaxed environment for the development of cloud computing. Second, the government should fine management, division of cloud security, provide hierarchical distinction between management measures. Finally, actively promote cooperation with other agencies, and the focus of demonstrations, from the point to the surface to promote national urban planning cloud computing industry [4].

Cryptographic processing and key update frequency. In the cloud computing process, we need to ensure data security but also need efficiency, and therefore, data encryption has become the first technology of choice to solve data security. Encryption is divided into symmetric encryption and asymmetric encryption, symmetric encryption, both parties use the same key to encrypt and decrypt data, which has high speed, high efficiency, the algorithm disclosed features, but the lack of security; asymmetric encryption keys Both sides use different keys, plus key into decryption key, although the algorithm complexity, but there are security. Therefore, when the cloud encryption, you should use symmetric encryption and asymmetric encryption combined, for a huge number of user data using symmetric encryption, for a small amount of data using symmetric encryption and asymmetric encryption method of combining the two key and secret text data stored in the cloud computing services, users save only asymmetric encryption key, so that you can effectively avoid management problems brought about by the same key symmetric encryption method, and help improve storage efficiency. For encryption technology, the key is the key. Therefore, we should pay attention to the update frequency key. The best way to have any complicated encryption security cycle, only a matter of time to crack the algorithm to solve this problem is to regularly update key. Furthermore, it should pay attention to the key update irregularities. If you frequently use the same key, easy to be illegal to crack; dangerous if only a few key update cycle, once find updated law, there is destruction.

The division level of privacy protection. The data stored in the cloud computing services contains many types, such as text, images, video, etc., for the user, different security levels of protection for each data type. And sensitized to the importance of regular and irregular time points: regular backups of data it is a cyclical data backup. Because companies need to arrange more staff and invest more funds in order to operate a variety of good growing backup data information. Therefore, from its own economic view, the company often set up a proper data backup time to meet the needs of their work. Generally speaking, the time schedule regular backups of computer data depends on computer databases within the data update cycle length and difficulty level recovery to the decision, if the cycle is shorter, recovery is difficult to shorten the backup time required, and vice versa can appropriate extend; data from time to time in accordance with the calculation A backup is a database within the backup data changes. If the computer database before creating new data or falsified data, the need for effective backup of data within the database information [5].

\section{The Computer Database Recovery Techniques}

The implementation of the database restore is mainly in order to build system database backups during normal operation of computer application system. This recovery technology is mainly in two ways: The first is based on reverse database system log recovery. The current database system operating practices used in general that contain the logging system, the so-called logging system, the 
aim of the operation of the database system, event sequence of activities and the amount of data in the system updates records. The main contents of the log of all transactions involve the database dealing technology and update processing and other markers. Under normal circumstances, a database transaction processing normal and abnormal termination submitted two results. Use the logging database technology enables database recovery back to the initial state; the second is based backup technology, computer database recovery techniques. The purpose of computer database backup technology is to ensure the integrity and security of database information to facilitate the smooth running database or computer system. Once a database failure, you need to re-enter the backup data. Intermediate between database backup and restore troubleshooting is independent, if the computer database backup and recovery technology to be the combination of technology, you can get a more complete and systematic troubleshooting computer database backup and recovery system to ensure stable operation of the computer. Once the application system put into official use, when a failure occurs can be combined with the actual situation by means of the local system or the Internet for remote restore database data.

\section{Conclusion}

In recent years, the term of cloud computing has been a hot spot and the commercial giants in computer industry have launched a lot of cloud computing products, more and more users are starting to use cloud computing services. However, the data security risks brought by current cloud computing services cannot be ignored. The cloud computing application technology faces huge crowd, so improve the data security of cloud computing services will be one of the topics of our long-term study.

\section{References}

[1] H.Q. Yan, P.K. Wang, The computer data security and recovery techniques, Consumer Electronics, 4(2013)

[2] Y.Q. Zhang, The data storage backup and disaster recovery, Computer CD Software and Applications, 12(2011)

[3] Q.Y. Zhang, The study of data security issue, Computer CD Software and Applications, 6(2012): 25-26.

[4] J. Lin, The research on data security based on cloud computing, Technology Square, 6(2013): 6-9.Study

[5] Y. Li, Study on cloud computing data access control method, Nanjing Posts University, 3(2013) 21-22. 